\title{
Manifolds with Ricci curvature in the Kato class: heat kernel bounds and applications
}

\author{
Christian Rose and Peter Stollmann ${ }^{1}$ \\ ${ }^{1}$ Technische Universität Chemnitz, Faculty of Mathematics, D - 09107 Chemnitz
}

June 16, 2021

\section{Introduction}

The 1973 paper by Tosio Kato entitled "Schrödinger operators with singular potentials", published in the Israel Journal of Mathematics [19], was meant to establish essential selfadjointness for Schrödinger operators under very mild restrictions on the potential term. Along the way, the author introduced two concepts that bear his name and turned out to be useful in different contexts. Actually, those two concepts, Kato's inequality and the Kato class of potentials, can be combined to give new insight in analysis and geometry of Riemannian manifolds, and this is, what the present survey is about.

We concentrate on the latter and record some of the implications that arise when the negative part of Ricci curvature obeys a Kato-type condition. Put very roughly, this is an application of methods from mathematical physics, more precisely, operator theory and Schrödinger operators, to questions about manifolds, namely, geometric properties that are related to the heat kernel. At the time being, papers concerning that topic are relatively recent and we have tried to record them all. If it should turn out that we missed a relevant paper, we would be grateful for references and include them in the future. Since ideas from two different communities are involved, we have decided to include some basics before stating the results.

In Section 1 we start by introducing the Kato class or Kato condition in a general set-up and explain its use in analysis and probability. The Kato condition can be seen as a condition of relative boundedness of a function (potential) $V$ with respect to some reference operator $H_{0}$ on the space in question. This reference operator was the usual Laplacian in $\mathbb{R}^{n}$ in the case of Kato's original paper and it will be the Laplace-Beltrami operator on a Riemannian manifold in the application that we have in mind. The Kato condition means that $V$ is, in a certain sense, small with respect to $H_{0}$ and that leads to the comforting fact that $H_{0}+V$ will inherit some of the "good" properties of $H_{0}$. In particular, mapping properties of the semigroup $\left(\mathrm{e}^{-t H_{0}}\right)_{t \geq 0}$ carry over to the perturbed semigroup $\left(\mathrm{e}^{-t\left(H_{0}+V\right)}\right)_{t \geq 0}$.

The next issue, also treated in the first Section, is the connection between heat kernel bounds for the Laplace-Beltrami operator and the validity of the Kato condition for functions in appropriate $L^{p}$-spaces. 
In Section 2 we give a short introductory account on domination of semigroups, a notion that is intimately connected with Kato's inequality and with the defining properties of Dirichlet forms in terms of the associated semigroups, known as the Beurling-Deny criteria. They allow for a pointwise comparison of the heat semigroup of the Laplace-Beltrami operator acting on functions and the heat semigroup of the Hodge-Laplacian acting on forms.

Throughout we will be concerned with a central point that makes the state of affairs somewhat complex. It is so important that we try to sketch it here and refer to the later sections for the technical details that we omit; we denote by $M$ a Riemannian manifold. The Hodge-Laplacian on 1-forms, denoted by $\Delta^{1}$ (here you see that our sign convention differs from the preferred one in mathematical physics), can be calculated by the Weitzenböck formula as

$$
\Delta^{1}=\nabla^{*} \nabla+\text { Ric }
$$

where the latter summand is considered as a matrix-valued function. Therefore, $\Delta^{1}$ itself looks like a Schrödinger operator, acting on vector-valued functions, though. Using Kato's inequality (introduced in that context by Hess, Schrader and Uhlenbrock in their paper [18]) the heat semigroup $\left(\mathrm{e}^{-t \Delta^{1}}\right)_{t \geq 0}$ of the Hodge-Laplacian is dominated by the semigroup of the Schrödinger operator

$$
\Delta+\rho \quad \text { on } L^{2}(M)
$$

where $\Delta=\Delta^{\mathrm{LB}}$ is the Laplace-Beltrami operator on functions and

$$
\rho(x):=\min \left\{\sigma\left(\operatorname{Ric}_{x}\right)\right\}, \quad x \in M,
$$

picks the smallest eigenvalue of the symmetric matrix $\mathrm{Ric}_{x}$ considered as an endomorphism of the space of 1 -forms.

Knowing that $\rho_{-}$, the negative part of $\rho$, is in some sense small with respect to $\Delta$, e.g., in terms of a Kato condition, allows one to control $\mathrm{e}^{-t(\Delta+\rho)}$ which in turn gives useful information on $\mathrm{e}^{-t \Delta^{1}}$ that can be used to estimate the first Betti number $b_{1}(M)$ in certain cases.

Now that looks like an easy lay-up but there is a very important drawback. The implications of the Kato condition are perturbation theoretic in spirit and require some smallness of $\rho_{-}$with respect to $\Delta$. But here, we cannot view $\rho$ as a perturbation of $\Delta$ in the usual sense, since we can not vary the potential $\rho$ independently of $\Delta$ : both depend on the Riemannian metric that defines the manifold!

The good news are that especially the Kato condition provides good quantitative estimates and so we can arrive at interesting consequences, provided that $\rho_{-}$satisfies a suitable Kato condition.

This work should be seen as part of a general program concerning geometric properties under curvature assumptions that are less restrictive than uniform bounds. Here, as well, an important comment is in order: in the compact case, all the quantities we consider depend quite regularly on the space variable. In particular, $\rho_{-}$is a pointwise minimum of smooth functions, hence continuous and, therefore, bounded. Hence, $\rho_{-}$is certainly in the Kato class, what would also be true if the $L^{p}$-norm 
of $\rho_{-}$for certain $p$ would be small enough in this case. However, $\rho_{-}$not only is relatively bounded, it is "really bounded". So why would one care about integrability conditions imposed on $\rho_{-}$or even the Kato condition? Well, the answer lies in the quantitative nature of our question and in the uniform control that is possible by assuming that, e.g., the $L^{p}$-norm of $\rho_{-}$for a family of metrics on $M$ obeys a suitable bound. We could, of course, use the infimum of $\rho$ as well but that would give much weaker estimates.

This being understood, we want to mention here the work of Gallot and coauthors in particular, who made important contributions in establishing analytic and geometric properties of Riemannian manifolds under the condition that the $L^{p_{-}}$ norm of $\rho_{-}$is small enough, see $[9,8,2,5$. Of course, the aforementioned program on geometric consequences of integral bounds on the Ricci curvature includes much more than the papers listed above, see, e.g., the original literature as well as 25 , 26, 24, 31] for more information.

A natural question that comes up is whether a Kato condition on $\rho_{-}$is sufficient to control the heat kernel. This was established by one of us in [27, building on an observation made in [36]. This is given in Section 3, where we also record similar results by Carron from 6 .

We used heat kernel bounds in relating $L^{p}$-properties and the Kato condition in order to prove upper bounds on $b_{1}(M)$ in [29] and to present conditions under which $b_{1}(M)=0$, generalizing earlier results by Elworthy and Rosenberg [7]. Actually, the latter reference was the starting point and main source of motivation for our above mentioned paper. Related work by different authors is collected in Section 4 . starting from Bochner's seminal work [4].

In Section 5 we mention some more consequences that arise from the control of the Kato property of Ricci curvature.

Acknowledgement: The second named author expresses his thanks to Daniel, Matthias and Radek for organizing such a wonderful conference and creating an atmosphere of open and respectful exchange of ideas.

\section{The Kato class and the Kato condition}

As mentioned in the introduction, the original definition of the property that defines the Kato class goes back to the celebrated paper [19] and was phrased as follows: the potential $V: \mathbb{R}^{n} \rightarrow \mathbb{R}$ is required to satisfy

$$
\lim _{r \rightarrow 0}\left[\sup _{x \in \mathbb{R}^{n}} \int_{|y| \leq r} V(x-y)|y|^{2-n} \mathrm{~d} y\right]=0,
$$

where we assume, in all that follows, that the space dimension satisfies $n \geq 3$ in order to avoid notational technicalities. Actually, an additional growth condition at 0 is present in Kato's paper. The important fact to notice is that condition (1) limits possible singularities, and uniformly so, in that $V$ is convolved with a singular kernel, in fact with the kernel of the fundamental solution of the Laplacian on $\mathbb{R}^{n}$ (up to a constant). We refer to [34, Section A2 in particular, for more details on 
the prehistory of the Kato condition; we wish to underline one important point and cite from the latter article, p. 453f., that "the naturalness of the Kato condition for $L^{p}$-properties was first noticed by Aizenman and Simon [1] in the path integral context (i.e., using the Feynman-Kac formula and Brownian motion) and by Agmon (cited as private communication in [34]) in the PDE context". Let us point out the following facts, which can be found in [34], where original references are given; we write $V \in \mathcal{K}_{n}$, and say that $V$ is in the Kato class, provided (1) holds.

Proposition 1.1. For $W \geq 0$ the following are equivalent:

(i) $W \in \mathcal{K}_{n}$,

(ii) $\left\|(-\Delta+\alpha)^{-1} W\right\|_{\infty} \rightarrow 0$ as $\alpha \rightarrow \infty$,

(iii) $\left\|\mathrm{e}^{-t(-\Delta-W)}\right\|_{\infty, \infty} \rightarrow 1$ as $t \rightarrow 0$.

See Theorem A.2.1 and Proposition A.2.3 in [34, p.454], which go back to [1]. The analytic properties in the latter Proposition were the starting point of a generalization of the Kato class given in [35]. There, the Laplacian is generalized to a selfadjoint operator $H_{0}$ on some $L^{2}(X, m)$ that is associated with a Dirichlet form (under some mild assumptions concerning $X$, see local citations for details). The fact that $H_{0}$ is associated with a Dirichlet form is equivalent to the fact that its semigroup is positivity preserving and contractive in the $L^{\infty}$-sense, in which case we speak of a Markovian semigroup.

In the latter article a Kato class of measures has been introduced and it has been shown that for this class $\hat{S}_{K}$ and $\mu \in \hat{S}_{K}, H_{0}-\mu$ can be defined by form methods and that the semigroup $\left(\mathrm{e}^{-t\left(H_{0}-\mu\right)}\right)_{t>0}$ shares many of the "good properties" of $\left(\mathrm{e}^{-t H_{0}}\right)_{t \geq 0}$. The main idea is that in order to control $L^{p}-L^{q}$-norms, e.g., of $\left(\mathrm{e}^{-t\left(H_{0}-\mu\right)}\right)_{t \geq 0}$ uniformly in $\mu=W \mathrm{~d} x$ for nice, say bounded $W \geq 0$, the relevant quantities are the following functions; as in [29] we omit the dependence on $H_{0}$ in the notation and set:

$$
c_{\text {Kato }}(W, \alpha):=\left\|\left(H_{0}+\alpha\right)^{-1} W\right\|_{\infty} \text { for } \alpha>0
$$

as well as

$$
b_{\text {Kato }}(W, \beta):=\int_{0}^{\beta}\left\|\mathrm{e}^{-t H_{0}} W\right\|_{\infty} \mathrm{d} t \quad \text { for } \beta>0 .
$$

As mentioned above, in most cases we are interested in dealing with bounded functions. In this case, the fact that the heat semigroup $\left(\mathrm{e}^{-t H_{0}}\right)_{t \geq 0}$ is Markovian implies that $\left\|\mathrm{e}^{-t H_{0}} W\right\|_{\infty}<\infty$ for $t>0$ as well as $\left\|\left(H_{0}+\alpha\right)^{-1} W\right\|_{\infty}<\infty$ for $\alpha>0$.

For general measurable $W \geq 0$ we can define

$$
c_{\text {Kato }}(W, \alpha):=\sup _{n \in \mathbb{N}}\left\|\left(H_{0}+\alpha\right)^{-1}(W \wedge n)\right\|_{\infty} \in[0, \infty]
$$

and say that $W$ satisfies a Kato condition, provided the latter quantity is finite for some $\alpha>0$.

The quantities above are related via functional calculus:

$$
\left(1-\mathrm{e}^{-\alpha \beta}\right) c_{\text {Kato }}(W, \alpha) \leq b_{\text {Kato }}(W, \beta) \leq \mathrm{e}^{\alpha \beta} c_{\text {Kato }}(W, \alpha),
$$


see [11], and we get that the behavior of $c_{\text {Kato }}(W, \alpha)$ for $\alpha \rightarrow \infty$ controls the behavior of $b_{\text {Kato }}(W, \beta)$ for $\beta \rightarrow 0$ and vice versa.

An important property is the stability of the boundedness of the semigroup in different $L^{p}$-spaces under Kato perturbations. It is implicit in the equivalence (i) $\Leftrightarrow$ (iii) of the above Proposition 1.1. The following explicit estimate goes back to [35. Theorem 3.3], and can be found in 29] in an equivalent dual form.

Proposition 1.2. Let $H_{0}$ be as above (the generator of a Markovian semigroup on $\left.L^{2}(X, m)\right)$ and $V \in L_{\text {loc }}^{1}(X)$ such that $b_{\text {Kato }}\left(V_{-}, \beta\right)=: b<1$ for some $\beta>0$. Then

$$
\left\|\mathrm{e}^{-t\left(H_{0}+V\right)}\right\|_{\infty, \infty} \leq \frac{1}{1-b} \mathrm{e}^{t \frac{1}{\beta} \log \frac{1}{1-b}} .
$$

Consequently, if ( $\left.\mathrm{e}^{-t H_{0}} ; t \geq 0\right)$ is ultracontractive, i.e., maps $L^{1}$ to $L^{\infty}$, then so is $\left(\mathrm{e}^{-t\left(H_{0}+V\right)} ; t \geq 0\right)$, a fact that can be deduced from the above and an interpolation argument, see [35, Theorem 5.1].

While we used an analytic set-up in the latter paper, one of the useful features of the Kato condition is that it is well suited for probabilistic techniques. So it is equally well possible to start from a given Markov process and define respective perturbations in a probabilistic manner. We refer to [1, 34] for the start and to [20, 21] for more recent contributions along these lines, as well as to [11] and the literature cited in these works.

One main point of interest here is to study the question whether the abstract version of a Kato condition as above, using quantities like $b_{\text {Kato }}, c_{\text {Kato }}$, can still be expressed in terms of kernels. In other words, whether a generalization of Proposition 1.1 holds true. The answer is yes, provided the heat kernel $p_{t}(\cdot, \cdot)$, the integral kernel of the heat semigroup, is controlled in some sense.

First of all, a very general condition for locally integrable functions to satisfy a Kato condition was given in 21 for general Markov processes associated to a Dirichlet form in $L^{2}(X, m)$ on a locally compact separable metric space $(X, d)$. The authors defined the Kato class relative to the Green kernel of the generator. For fixed $\nu \geq \beta>0$, a non-negative function $V \in L_{\text {loc }}^{1}(X, m)$ belongs to this Kato class $K_{\nu, \beta}(X)$ if

$$
\lim _{r \rightarrow 0} \sup _{x \in X} \int_{B(x, r)} G(x, y) V(y) \mathrm{d} m(y)=0,
$$

where $G(x, y):=G(d(x, y))$ with $G(r)=r^{\beta-\nu}$ and $B(x, r)$ denotes the metric ball around $x \in X$ of radius $r>0$. Denote by $L_{\text {unif }}^{p}(X)$ the set of functions $f$ such that

$$
\sup _{x \in X} \int_{B(x, 1)}|f|^{p} \mathrm{~d} m<\infty .
$$

Theorem 1.3 ([21, Theorem 3.3]). For all $\nu \geq \beta>0, p>\nu / \beta$, we have

$$
f \in L_{\text {unif }}^{p}(X) \quad \Rightarrow \quad|f| \in K_{\nu, \beta}(X)
$$

provided there is a positive increasing function $V$ on $(0, \infty)$ such that $r \mapsto V(r) / r^{\nu}$ is increasing or bounded and $\sup _{x \in X} m(B(x, r)) \leq V(r)$ for all $r>0$, and the heat 
kernel satisfies upper and lower bounds in the following way: there exist two positive increasing functions $\varphi_{1}, \varphi_{2}:(0, \infty) \rightarrow(0, \infty)$ such that

$$
\int_{1}^{\infty} \frac{1}{t} \max \left\{V(t), t^{\nu}\right\} \varphi_{2}(t) \mathrm{d} t<\infty
$$

and for any $x, y \in X, t \in\left(0, t_{0}\right]$, we have

$$
\frac{1}{t^{\nu / \beta}} \varphi_{1}\left(\frac{d(x, y)}{t^{1 / \beta}}\right) \leq p_{t}(x, y) \leq \frac{1}{t^{\nu / \beta}} \varphi_{2}\left(\frac{d(x, y)}{t^{1 / \beta}}\right) .
$$

More specifically, in the case of a geodesically complete Riemannian manifold with bounded geometry, bounds on the heat kernel are explicit, leading to the following.

Theorem 1.4 ([11, Theorem 2.9]). Let $M$ be a geodesically complete Riemannian manifold of dimenion $n \geq 2$ with Ricci curvature bounded below and assume that there are $C, R>0$ such that for all $x \in M$ and $r \in(0, R]$, one has

$$
\operatorname{Vol}(B(x, r)) \geq C r^{n} .
$$

Then we have

(i) $V \in K_{n, 2}(M)$ if and only if

$$
\lim _{t \rightarrow 0} \sup _{x \in M} \int_{0}^{t} p_{t}(x, y)|V(y)| \operatorname{dvol}(y)=0 .
$$

(ii) for any $p>n / 2$, we have $L_{\text {unif }}^{p}(M) \subset K_{n, 2}(M)$.

In particular, [11, Corollary 2.11] then gives $L^{p}(M)+L^{\infty}(M) \subset K_{n, 2}(M)$ under the same assumptions. The non-collapsing of the volume of the balls seems strong, but can only be avoided by replacing it by a lower bound on the heat kernel, a condition that is stronger than the volume bound.

Theorem 1.5 ([14, Proposition 3.2]). Let $M$ be a Riemannian manifold of dimension $n \geq 2$ and $p>n / 2$.

(i) If there are $C, t_{0}>0$ such that for all $t \in\left(0, t_{0}\right]$ and all $x \in M$ we have $p_{t}(x, x) \leq C t^{-n / 2}$, then, for any $V \in L^{p}(M)+L^{\infty}(M)$,

$$
\lim _{t \rightarrow 0} \sup _{x \in M} \int_{0}^{t} p_{t}(x, y)|V(y)| \operatorname{dvol}(y)=0 .
$$

(ii) Let $M$ be geodesically complete and assume that there are positive constants $C_{1}, \ldots, C_{6}, t_{0}>0$ such that for all $t \in\left(0, t_{0}\right], x, y \in M, r>0$ one has $\operatorname{Vol}(B(x, r)) \leq C_{1} r^{n} \mathrm{e}^{C_{2} r}$ and

$$
C_{3} t^{-n / 2} \mathrm{e}^{-C_{4} \frac{d(x, y)^{2}}{t}} \leq p_{t}(x, y) \leq C_{5} t^{-n / 2} \mathrm{e}^{-C_{6} \frac{d(x, y)^{2}}{t}} .
$$

Then, one has

$$
L_{\text {unif }}^{p}(M)+L^{\infty}(M) \subset K_{n, 2}(M) .
$$


In the special case of compact manifolds, the potentials in the Kato class can be characterized with the help of uniform heat kernel estimates, i.e., there are some constants $C, k, t_{0}>0$ such that

$$
\forall x, y \in M, t \in\left(0, t_{0}\right]: \quad p_{t}(x, y) \leq C t^{-k} .
$$

Classically, such estimates follow from so-called isoperimetric inequalities under certain assumptions on the Ricci curvature. In [29], the authors exhibited the neccessary analytic framework based on [8], leading to the following:

Proposition 1.6 ([29, Theorem 4.1]). Let $D>0$ and $q>n \geq 3$. There is an explicit constant $\varepsilon>0$ such that for any compact Riemannian manifold $M$ with $\operatorname{dim} M=n, \operatorname{diam}(M) \leq D$, and $\left\|\rho_{-}\right\|_{q / 2}<\varepsilon$, for any $p>q / 2$ there is $C>0$ such that for any $0 \leq V \in L^{p}(M)$ we have

$$
c_{\text {Kato }}(V, \alpha) \leq C\|V\|_{p} \int_{0}^{\infty} \mathrm{e}^{-\alpha t} \max \left\{1, t^{-q / 2 p}\right\} \mathrm{d} t .
$$

An analogous estimate also holds for $b_{\text {Kato }}(V, \beta)$ by a direct computation or by using the relation (2). Due to the fact that the decay rate $k$ of the heat kernel in (3) depends on the integrability of the negative part of the Ricci curvature, the integral on the right-hand side only converges for potentials with a higher integrability.

\section{Domination of semigroups, Kato's inequality and comparison for the heat semigroup on functions and on 1-forms}

We start with some historical remarks and with the famous Kato's inequality that reads

$$
\Delta|u| \leq \Re(\operatorname{sgn} \bar{u}) \Delta u
$$

according to our sign convention. Actually, in its original form as Lemma A in [19], magnetic fields were included on the left-hand side. We should note, in passing, that (4) is meant in the distributional sense and it is assumed that $\Delta u \in L_{\mathrm{loc}}^{1}$. The interesting feature of (4) is that it can be expressed equivalently in terms of the following positivity property for the semigroup:

$$
\left|\mathrm{e}^{-t \Delta} f\right| \leq \mathrm{e}^{-t \Delta}|f|, \quad\left(f \in L^{2}, t \geq 0\right)
$$

which can be seen as the property that the heat semigroup dominates itself. To explain that, we follow the paper [17] in introducing the neccessary concepts. See also [32, 33, 18, as well as [22] and the literature cited there for more recent contributions. The absolute value $|f|$ is replaced by a more general mapping, allowing vector valued functions. We will use some terminology without explanation. All the neccessary facts can be found in the articles above.

We start with a real Hilbert space $\mathcal{K}$ and a cone $\mathcal{K}^{+}$that is compatible with the inner product $\langle\cdot, \cdot\rangle$. Given another, real or complex, Hilbert space $\mathcal{H}$, a map $|\cdot|: \mathcal{H} \rightarrow \mathcal{K}^{+}$ is called an absolutely pairing map, provided the following properties hold (we write $\langle\cdot, \cdot\rangle$ for both inner products in a slight abuse of notation): 
- $\forall f_{1}, f_{2} \in \mathcal{K}: \quad\left|\left\langle f_{1}, f_{2}\right\rangle\right| \leq\left\langle\left|f_{1}\right|,\left|f_{2}\right|\right\rangle$,

- $\forall f \in \mathcal{H}: \quad\langle f, f\rangle=\langle|f|,|f|\rangle$,

- $\forall g \in \mathcal{K}^{+} \exists f_{2} \in \mathcal{H}: \quad\left|f_{2}\right|=g$ and $\forall f_{1} \in \mathcal{H}: \quad\left\langle f_{1}, f_{2}\right\rangle=\left\langle\left|f_{1}\right|, g\right\rangle$.

Two elements satisfying the third condition are called absolutely paired. Given an absolutely pairing map, we can talk about domination of operators that act on $\mathcal{K}$ and $\mathcal{H}$ respectively. First, however, we give the example that is important for us here:

$$
\mathcal{K}=L^{2}(M), \quad \mathcal{H}=L^{2}\left(M, \Omega^{1}\right),
$$

the latter consisting of square integrable sections of the cotangent bundle, where the forms $\omega(x)$ are measured in terms of the inner product induced by the Riemannian metric, written as $|\omega(x)|$. It is not hard to see that

$$
|\cdot|: L^{2}\left(M, \Omega^{1}\right) \rightarrow L^{2}(M), \quad \omega \mapsto|\omega(\cdot)|
$$

is an absolutely pairing map; of course, both $L^{2}$-spaces are built upon the Riemannian volume.

Going back to the general case we can say that a bounded linear operator $A$ on $\mathcal{H}$ is dominated by a bounded linear operator $B$ on $\mathcal{K}$, provided

$$
|A f| \leq B|f| \quad(f \in \mathcal{H}) .
$$

Clearly, this notion depends on the absolute map $|\cdot|$. The following powerful characterization of semigroup domination can be found in [17, Theorem 2.15]. Let $H$ and $K$ be (the negative of) generators of the strongly continuous semigroups $T_{t}=\mathrm{e}^{-t H}$ and $S_{t}=\mathrm{e}^{-t K}$.

Theorem 2.1. In the situation above, the following statements are equivalent:

(i) $\left(T_{t}\right)_{t \geq 0}$ is dominated by $\left(S_{t}\right)_{t \geq 0}$, i.e.,

$$
\left|T_{t} f\right| \leq S_{t}|f| \quad(f \in \mathcal{H}) .
$$

(ii) $H$ and $K$ satisfy a generalized Kato's inequality: For all $f_{1} \in \operatorname{dom}(H)$ and $f_{2} \in \mathcal{H}$ such that $\left|f_{2}\right| \in \operatorname{dom}\left(K^{*}\right)$ and $f_{1}$ and $f_{2}$ absolutely paired:

$$
\Re\left\langle H f_{1}, f_{2}\right\rangle \geq\left\langle\left|f_{1}\right|, K^{*}\left|f_{2}\right|\right\rangle .
$$

Let us mention that condition (ii) has a counterpart in a version of the first Beurling-Deny criterion, see [33] and [22]. The comforting fact for us is that $\left(\mathrm{e}^{-t \bar{\Delta}}\right)_{t \geq 0}$ and $\left(\mathrm{e}^{-t \Delta}\right)_{t \geq 0}$ enjoy the domination relation aluded to above, where $\bar{\Delta}=\nabla^{*} \nabla$ is the Bochner-Laplacian. This has been proven in terms of a Kato inequality by Hess, Schrader and Uhlenbrock in [18], so that

$$
\left|\mathrm{e}^{-t \bar{\Delta}} \omega\right| \leq \mathrm{e}^{-t \Delta}|\omega|
$$


for all $t \geq 0$ and $\omega \in L^{2}\left(M, \Omega^{1}\right)$. The Weitzenböck formula and abstract results on sums of generators give

$$
\left|\mathrm{e}^{-t \Delta^{1}} \omega\right| \leq \mathrm{e}^{-t(\Delta+\rho)}|\omega|
$$

and, moreover,

$$
\operatorname{Tr}\left(\mathrm{e}^{-t \Delta^{1}}\right) \leq n \operatorname{Tr}\left(\mathrm{e}^{-t(\Delta+\rho)}\right) .
$$

Both these inequalities have geometrical implications, as we already mentioned above and as we will see in more detail in Section 4 below.

We mention in passing that domination of semigroups can also be treated probabilistically, cf. [30]. This gives a nice pointwise estimate on the heat kernels, as shown in Theorem 3.5 of the latter paper by Rosenberg, which we state here in the special case under consideration. For this reason, denote by $p_{t}^{(1)}(x, y)$ the heat kernel of the Hodge-Laplacian $\Delta^{(1)}$ and $p_{t}^{(0)}(x, y)$ the heat kernel of $\Delta+\rho$ acting on functions.

Theorem 2.2 ([30, Theorem 3.5]). For all $t>0, x, y \in M$, we have

$$
\left|p_{t}^{(1)}(x, y)\right| \leq n p_{t}^{(0)}(x, y)
$$

\section{The Kato condition implies heat kernel bounds}

Proofs of the fact mentioned in the title of this section are based on the following result by Qi S. Zhang and M. Zhu:

Theorem 3.1 ([36, Theorem 1.1]). Let $M$ be a compact Riemannian manifold of dimension $n$, and $u$ a positive solution to the heat equation

$$
\partial_{t} u=-\Delta u
$$

Suppose that either one of the following conditions holds:

(i) $\left\|\rho_{-}\right\|_{p}<\infty$ for $p>n / 2$, and that there is a $c>0$ such that for all $x \in M$ and $r \in(0,1]$, we have $\operatorname{Vol}(B(x, r)) \geq c r^{n}$.

(ii) $\sup _{x \in M} \int_{M} \rho_{-}^{2}(y) d(x, y)^{2-n} \operatorname{dvol}(y)<\infty$ and the heat kernel is bounded from above.

Then, for any $\alpha \in(0,1)$, there are an explicit continuous function $J:(0, \infty) \rightarrow(0,1)$ and $c>0$ such that

$$
J(t) \frac{|\nabla u|^{2}}{u^{2}}-\frac{\partial_{t} u}{u} \leq \frac{c}{J(t) t}, \quad(t \in(0, \infty)) .
$$

An inequality of the type (5) yields an explicit upper bound of the heat kernel by a nowadays standard technique introduced by Li and Yau in [23]. A thorough inspection of the reasoning in [36] shows that the Kato condition on $\rho_{-}$indeed implies heat kernel estimates in the following sense: 
Theorem 3.2 ([27]). Let $n \geq 3$ and $\beta>0$. For any closed Riemannian manifold $M$ of dimension $n$ satisfying $\operatorname{diam}(M) \leq \sqrt{\beta}$, and

$$
b:=b_{\text {Kato }}\left(\rho_{-}, \beta\right)<\frac{1}{16 n},
$$

there are explicit constants $C=C(n, b, \beta)>0$ and $\kappa=\kappa(n, b, \beta)>0$ such that we have

$$
p_{t}(x, y) \leq \frac{C}{\operatorname{Vol}(M)} t^{-\kappa} \quad\left(t \in\left(0, \beta^{2} / 4\right], x, y \in M\right) .
$$

A different version that is more explicit in the sense that it fits well with the euclidean case was obtained independently by G. Carron. For its formulation we use the notation of the present paper:

Theorem $3.3([6])$. There is a constant $c_{n}$ depending on $n$ alone with the following property: Let $D:=\operatorname{diam}(M)$ and $T$ the largest time such that

$$
b_{\text {Kato }}\left(\rho_{-}, T\right) \leq \frac{1}{16 n} .
$$

Then

$$
p_{t}(x, x) \leq \frac{c_{n}}{\operatorname{Vol}(B(x, \sqrt{t}))} \quad(t \in(0, T / 2], x \in M) .
$$

See Corollary 3.9 in [6] and Section 3 of the latter article. Note that the results above are closely related via the so-called volume doubling condition. [6, Proposition 3.8] also shows that the volume doubling condition is indeed satisfied under the curvature assumptions of the above theorems. See also [28] for the connection.

\section{Bounds on the first Betti number}

The first Betti number, $b_{1}(M)$, is a tool for classifying compact Riemannian manifolds $M$ of dimension $n$. By definition, $b_{1}(M)$ is the dimension of the first cohomology group, $b_{1}(M):=\operatorname{dim} \mathcal{H}^{1}(M)$, where $\mathcal{H}^{1}(M)$ is the real linear space quotient of the closed differential 1-forms on $M$ by the exact forms. This group describes in some sense the $(n-1)$-dimensional holes of $M$ and is therefore actually of topological nature, clarifiying its relevance for the classification of manifolds. Bochner was the first to observe that it is quite easy to derive bounds on $b_{1}(M)$ if the Ricci tensor is non-negative or even positive in some point of $M$. More precisely, he showed in [4] the following theorem, although, the result is not explicitely stated in the form below:

Theorem 4.1 (Bochner '46). Let $M$ be a compact Riemannian manifold of dimension $n$. If the Ricci curvature is non-negative, then

$$
b_{1}(M) \leq n .
$$

If, additionally, there is a point in $M$ such that the Ricci curvature is strictly positive at that point, then

$$
b_{1}(M)=0
$$


Actually, the above theorem follows implicitly from the Weitzenböck formula

$$
\Delta^{1}=\bar{\Delta}+\text { Ric },
$$

where $\Delta^{1}$ is the Hodge-Laplacian acting on one-forms, $\bar{\Delta}:=\nabla^{*} \nabla$ the so-called rough or Bochner-Laplacian, and Ric denotes the Ricci tensor interpreted as a section of endomorphisms on the space of one forms as above. Any equivalence class in $\mathcal{H}^{1}(M)$ can be represented by a harmonic one-form, such that

$$
\operatorname{dim} \operatorname{ker}\left(\Delta^{1}\right)=\operatorname{dim} \mathcal{H}^{1}(M) .
$$

Using the non-negativity of Ric in quadratic form sense implies directly that there are only parallel forms in $\operatorname{ker}\left(\Delta^{1}\right)$, which vanish under the additional positivity assumption on Ric. This classical ansatz, known as the first application of the nowadays called Bochner method, seems not to lead to results allowing some negative Ricci curvature. However, instead of a geometric argument it is possible to use form methods to control the kernel of $\Delta^{1}$. The main observation here can be found in [7] by Elworthy and Rosenberg building on the domination property established by Hess, Schrader and Uhlenbrock in [18, discussed in Section 2 above: namely, for any square integrable section of one-forms $\omega \in L^{2}\left(M ; \Omega^{1}\right)$, we have:

$$
\left|\mathrm{e}^{-t \Delta^{1}} \omega\right| \leq \mathrm{e}^{-t(\Delta+\rho)}|\omega|,
$$

where the norms above are taken pointwise in the cotangent bundle of $M$. If $\omega$ is harmonic, the left-hand side equals $|\omega|$. If the semigroup on the right-hand side is generated by a positive operator $\Delta+\rho>0$, we can let $t \rightarrow \infty$, so that $\mathrm{e}^{-t(\Delta+\rho)}|\omega| \rightarrow 0$ which gives $\omega=0$, yielding a method to conclude the triviality of $\operatorname{ker}\left(\Delta^{1}\right)$. The issue here is that we cannot easily treat $\rho$ as a perturbation of $\Delta$ since both of them depend on the metric tensor of $M$. Therefore, it is not trivial to get positivity of the operator $\Delta+\rho$.

A general strategy is to control the part of the Ricci curvature lying below a certain positive threshold. Elworthy and Rosenberg derived the following theorem along these lines with a more complicated method of proof based on Sobolev embedding theorems and eigenfunction estimates:

Theorem 4.2 (Elworthy/Rosenberg '91). Let $M$ be a compact manifold, $X \subset M$, $K, K_{0}>0$, Ric $\geq-K_{0}$ on $X$, Ric $\geq K$ on $M \backslash X$. There exists $a>0$ depending on the quantities above such that if

$$
\operatorname{Vol}(X)<a \operatorname{Vol}(M)
$$

then $b_{1}(M)=0$.

Unfortunately, the constant $a$ in the above theorem is far from being explicit and it also still depends on the lower bound $K$ for the Ricci tensor.

When Elworthy and Rosenberg published their article there was already a result that implies the assertion in the latter theorem in Gallot's article [8] from 1988. In fact, Gallot proved an estimate of the first eigenvalue of $\Delta+V$ for some potential $V$ 
in terms of its $L^{p}$-norm, so that (8) leads to the vanishing of $b_{1}(M)$; we also mention [3] in which the same basic idea is nicely explained in a little more restrictive context.

Rosenberg and Yang also recognized that integral bounds are the right thing to look for and arrived at the following result, Theorem 4 in [31]:

Theorem 4.3 (Rosenberg/Yang '94). Let $M$ be an n-dimensional complete Riemannian manifold. Assume that there exist constants $A, B>0$ such that for any $f \in C_{c}^{\infty}(M)$

$$
\left(\int_{M}|f(x)|^{\frac{2 n}{n-2}} \mathrm{~d} \operatorname{vol}(x)\right)^{\frac{n-2}{n}} \leq A \int_{M}|\nabla f(x)|^{2} \operatorname{dvol}(x)+B \int_{M}|f(x)|^{2} \operatorname{dvol}(x) .
$$

Then, whenever for some $\rho_{0}>0$,

$$
\left\|\left(\rho-\rho_{0}\right)_{-}\right\|_{\frac{n}{2}}<\min \left\{A^{-1}, \rho_{0} B^{-1}\right\},
$$

it follows that $b_{1}(M)=0$.

The main idea is to decompose

$$
\Delta+\rho=\Delta+\rho_{0}+\left(\rho-\rho_{0}\right) \geq \Delta+\rho_{0}-\left(\rho-\rho_{0}\right)_{-},
$$

which is positive as soon as $\left(\rho-\rho_{0}\right)_{-}$is relatively bounded w. r. t. $\Delta$ for some formbound smaller than one and such an estimate can be deduced from a Sobolev embedding theorem. The nice fact about the latter result is that it allows a statement in the threshold case $\frac{n}{2}$ as far as integrability of $\left(\rho-\rho_{0}\right)_{-}$is concerned. Moreover, the argument is quite direct and doesn't rely on explicit heat kernel estimates, an issue we turn to next.

Assuming the semigroup generated by $\Delta$ is ultracontractive, i.e., there are constants $C, k, t_{0}>0$ such that

$$
\left\|\mathrm{e}^{-t \Delta}\right\|_{1, \infty} \leq C t^{-k}, \quad t \in\left(0, t_{0}\right),
$$

perturbation techniques based on the Kato condition lead to quantitative results as well. With the decomposition of $\Delta+\rho$ as above, the assumption of ultracontractivity allows to handle $\left(\rho-\rho_{0}\right)_{-}$as a Kato-perturbation, that means, we are looking for conditions that give

$$
b_{\text {Kato }}\left(\left(\rho-\rho_{0}\right)_{-}, \rho_{0}^{-1}\right):=\int_{0}^{\rho_{0}^{-1}}\left\|\mathrm{e}^{-t \Delta}\left(\rho-\rho_{0}\right)_{-}\right\|_{\infty} \mathrm{d} t<1 .
$$

Ultracontractivity of the heat semigroup also implies its continuity from $L^{p}(M)$ to $L^{\infty}(M)$ for all $p \in(0, \infty]$, so that

$$
b_{\text {Kato }}\left(\left(\rho-\rho_{0}\right)_{-}, \rho_{0}^{-1}\right) \leq C\left\|\left(\rho-\rho_{0}\right)_{-}\right\|_{p} \int_{0}^{\rho_{0}^{-1}} t^{-p / k} \mathrm{~d} t<1
$$

if $p<k$ and the $L^{p}$-norm on the right-hand side is small enough. This explicitly computable quantity led to the result below. 
Theorem 4.4 (Rose/Stollmann '17). Let $n \in \mathbb{N}, n \geq 3, p>n / 2, D>0$. There is an explicitly computable $\varepsilon>0$ such that for all compact Riemannian manifolds $M$ of dimension $n, \operatorname{diam}(M) \leq D$, and

$$
\frac{1}{\operatorname{Vol}(M)} \int_{M} \rho_{-}^{p} \mathrm{dvol}<\varepsilon
$$

we have $b_{1}(M)=0$.

At the heart of the proof lies a deep isoperimetric estimate from [8] that holds under the assumption that the averaged $L^{p}$-norm of the Ricci curvature is small enough, implying ultracontractivity of the heat semigroup.

We now turn to the question whether it is enough to assume smallness of the Kato constant $b_{\text {Kato }}$ to derive bounds on $b_{1}(M)$. The main oberservation is

$$
\operatorname{dim} \operatorname{ker}\left(\Delta^{1}\right) \leq \operatorname{Tr}\left(\mathrm{e}^{-t \Delta^{1}}\right) \leq n \operatorname{Tr}\left(\mathrm{e}^{-t\left(\Delta+\rho_{-}\right)}\right) \leq n \operatorname{Vol}(M)\left\|\mathrm{e}^{-t\left(\Delta+\rho_{-}\right)}\right\|_{1, \infty},
$$

so that we get bounds on $b_{1}(M)$ as soon as we can control $\left\|\mathrm{e}^{-t\left(\Delta+\rho_{-}\right)}\right\|_{1, \infty}$. The ultracontractivity estimate is crucial here as well as the stability of ultracontractivity under Kato-class perturbations, stated in Proposition 1.2 above.

A little work and putting everything together yields

Theorem 4.5 (Rose/Stollmann '17). $3 \leq n<p<2 q$ and $D>0$. There is an $\varepsilon>0$ and a constant $K(p)>0$ depending only on $p$ such that for all compact Riemannian manifolds $M$ with $\operatorname{dim} M=n$, and $\operatorname{diam}(M) \leq D$ with $\left\|\rho_{-}\right\|_{q} \leq \varepsilon$, we have

$$
b_{1}(M) \leq n \cdot\left(\frac{2}{1-\varepsilon^{-1}\left\|\rho_{-}\right\|_{p}}\right)^{2 \frac{1+\varepsilon^{-1}\left\|\rho_{-}\right\|_{p}}{1-\varepsilon^{-1}\left\|\rho_{-}\right\|_{p}}+\frac{p}{2}}\left(1+K(p) D^{\frac{p}{2}}\right) .
$$

Even though the Kato condition on the part of Ricci curvature below a positive level is sufficient to obtain the triviality of $\mathcal{H}^{1}(M)$ we do not know yet whether a Kato-bound on the negative part of Ricci curvature implies a non-trivial bound on $b_{1}(M)$. The ultracontractivity is a neccessary assumption such that equation (10) can be applied and calculated. Fortunately, Theorem 3.2 above shows that the smallness of $b_{\text {Kato }}\left(\rho_{-}, \beta\right)$ for some $\beta>0$ implies a heat kernel upper bound, giving the desired ultracontractive bound and in turn the bound on the first Betti number.

Theorem 4.6 (Rose '17). Let $n \geq 2$ and $\beta>0$. Any compact Riemannian manifold with $\operatorname{dim} M=n$, $\operatorname{diam} M \leq \sqrt{\beta}$, and

$$
b:=b_{\text {Kato }}\left(\rho_{-}, \beta\right) \leq \frac{1}{16 n},
$$

satisfies

$$
b_{1}(M) \leq n \cdot\left(\frac{2}{1-b}\right)^{\left(1+\frac{1}{\beta}\right) \frac{1+b}{1-b}+\frac{1}{n-1}} \mathrm{e}^{\frac{3 n}{n-1}} .
$$

Additionally, Carron showed in [6], that a clever improvement of the upper bound of the heat kernel and Gromov's trick lead to the following estimate.

Theorem 4.7 (Carron '16). Let $n \geq 2$ and $\beta>0$. There is an $\varepsilon>0$ such that any compact Riemannian manifold with $\operatorname{diam} M \leq \sqrt{\beta}$ and $b_{\text {Kato }}\left(\rho_{-}, \beta\right)<\varepsilon$ satisfies $b_{1}(M) \leq n$. 


\section{Concluding remarks}

Here we first briefly mention some other results that have been obtained under the assumption that the negative part of Ricci curvature satisfies a Kato condition.

We already heavily cited [6] above. Apart from what we already referred, Carron shows, amongst other things and assumptions, that such a curvature condition allows to control the volume growth of balls from above, giving volume doubling and an upper bound on the volume of balls that coincides with the euclidean case.

In [13], the authors extend the heat semigroup characterization of functions of bounded variation to manifolds whose Ricci curvature is not necessarily bounded below, again assuming that the negative part of Ricci curvature satisfies a Kato condition.

There is also a big distribution by Güneysu, who extended the concepts of Kato class potentials to the context of vector-valued functions on manifolds, see, e.g., [15, 16, 11, 10, 12] and the cited literature therein.

Let us end with a meta question: While it is by now quite well understandable that Kato conditions on the negative part of Ricci curvature can be used to find bounds on $b_{1}(M)$ as we hopefully convinced our readers above, there is still some kind of mystery in the fact, that Kato class Ricci curvature actually leads to heat kernel bounds and other geometric features. In fact, for the former results, one uses domination and a Schrödinger operator that features $\rho_{-}$as a potential term. For the latter case, however, the operator in question is the Laplace-Beltrami operator itself that exhibits no potential term.

Apart from the obvious fact that the proofs work: why is it true? A better understanding is certainly needed, e.g., for an extension of some of the results we mentioned to the non-compact case.

\section{References}

[1] M. Aizenman and B. Simon. Brownian motion and Harnack inequality for Schrödinger operators. Comm. Pure Appl. Math., 35(2):209-273, 1982.

[2] P. H. Bérard. From vanishing theorems to estimating theorems: the Bochner technique revisited. Bull. Amer. Math. Soc. (N.S.), 19(2):371-406, 1988.

[3] P.H. Bérard. A lower bound for the least eigenvalue of $\Delta+V$. Manuscripta Math., 69(3):255-259, 1990.

[4] S. Bochner. Vector fields and Ricci curvature. Bull. Amer. Math. Soc., 52:776797, 1946.

[5] P Bérard and G Besson. Number of bound states and estimates on some geometric invariants. Journal of Functional Analysis, 94(2):375 - 396, 1990.

[6] G. Carron. Geometric inequalities for manifolds with Ricci curvature in the Kato class. 2016. https://arxiv.org/abs/1612.03027 [math.DG]. 
[7] K. D. Elworthy and S. Rosenberg. Manifolds with wells of negative curvature. Invent. Math., 103(3):471-495, 1991. With an appendix by Daniel Ruberman.

[8] S. Gallot. Inégalités isopérimétriques et analytiques sur les variétés riemanniennes. Astérisque, (163-164):5-6, 31-91, 281 (1989), 1988. On the geometry of differentiable manifolds (Rome, 1986).

[9] S. Gallot. Isoperimetric inequalities based on integral norms of Ricci curvature. Astérisque, (157-158):191-216, 1988. Colloque Paul Lévy sur les Processus Stochastiques (Palaiseau, 1987).

[10] B. Güneysu. On the Feynman-Kac formula for Schrödinger semigroups on vector bundles. Dissertation, Rheinische Friedrich-Wilhelms-Universität Bonn, 2010.

[11] B. Güneysu. Kato's inequality and form boundedness of Kato potentials on arbitrary Riemannian manifolds. Proc. Amer. Math. Soc., 142(4):1289-1300, 2014.

[12] B. Güneysu. Covariant Schrödinger semigroups on noncompact Riemannian manifolds. Habilitation, Humboldt-Universität zu Berlin, 2016.

[13] B. Güneysu and D. Pallara. Functions with bounded variation on a class of Riemannian manifolds with Ricci curvature unbounded from below. Math. Ann., 363(3-4):1307-1331, 2015.

[14] B. Güneysu and O. Post. Path integrals and the essential self-adjointness of differential operators on noncompact manifolds. Math. Z., 275(1-2):331-348, 2013.

[15] Batu Güneysu. On generalized Schrödinger semigroups. J. Funct. Anal., 262(11):4639-4674, 2012.

[16] Batu Güneysu. Heat kernels in the context of Kato potentials on arbitrary manifolds. Potential Anal., 46(1):119-134, 2017.

[17] H. Hess, R. Schrader, and D. A. Uhlenbrock. Domination of semigroups and generalization of Kato's inequality. Duke Math. J., 44(4):893-904, 1977.

[18] H. Hess, R. Schrader, and D. A. Uhlenbrock. Kato's inequality and the spectral distribution of Laplacians on compact Riemannian manifolds. J. Differential Geom., 15(1):27-37 (1981), 1980.

[19] T. Kato. Schrödinger operators with singular potentials. In Proceedings of the International Symposium on Partial Differential Equations and the Geometry of Normed Linear Spaces (Jerusalem, 1972), volume 13, pages 135-148 (1973), 1972 .

[20] K. Kuwae and M. Takahashi. Kato class functions of Markov processes under ultracontractivity. In Potential theory in Matsue, volume 44 of Adv. Stud. Pure Math., pages 193-202. Math. Soc. Japan, Tokyo, 2006. 
[21] K. Kuwae and M. Takahashi. Kato class measures of symmetric Markov processes under heat kernel estimates. J. Funct. Anal., 250(1):86-113, 2007.

[22] D. Lenz, M. Schmidt, and M. Wirth. Domination of quadratic forms. ArXiv e-prints, November 2017.

[23] P. Li and S.-T. Yau. On the parabolic kernel of the Schrödinger operator. Acta Math., 156(3-4):153-201, 1986.

[24] P. Petersen and C. Sprouse. Integral curvature bounds, distance estimates and applications. J. Differential Geom., 50(2):269-298, 1998.

[25] P. Petersen and G. Wei. Relative volume comparison with integral curvature bounds. Geom. Funct. Anal., 7(6):1031-1045, 1997.

[26] P. Petersen and G. Wei. Analysis and geometry on manifolds with integral Ricci curvature bounds. II. Trans. Amer. Math. Soc., 353(2):457-478, 2001.

[27] C. Rose. Li-Yau gradient estimate for compact manifolds with negative part of Ricci curvature in the Kato class. 2016. https://arxiv.org/abs/1608.04221 [math.DG].

[28] C. Rose. Heat kernel estimates based on Ricci curvature integral bounds. Dissertation, Technische Universität Chemnitz, 2017.

[29] C. Rose and P. Stollmann. The Kato class on compact manifolds with integral bounds of Ricci curvature. to appear in P. Am. Math. Soc., arXiv:1601.07441 [math.DG], 2016.

[30] S. Rosenberg. Semigroup domination and vanishing theorems. In Geometry of random motion (Ithaca, N.Y., 1987), volume 73 of Contemp. Math., pages 287-302. Amer. Math. Soc., Providence, RI, 1988.

[31] S. Rosenberg and D. Yang. Bounds on the fundamental group of a manifold with almost nonnegative Ricci curvature. J. Math. Soc. Japan, 46(2):267-287, 1994.

[32] B. Simon. An abstract Kato's inequality for generators of positivity preserving semigroups. Indiana Univ. Math. J., 26(6):1067-1073, 1977.

[33] B. Simon. Kato's inequality and the comparison of semigroups. J. Funct. Anal., 32(1):97-101, 1979.

[34] B. Simon. Schrödinger semigroups. Bull. Amer. Math. Soc. (N.S.), 7(3):447$526,1982$.

[35] P. Stollmann and J. Voigt. Perturbation of Dirichlet forms by measures. Potential Anal., 5(2):109-138, 1996.

[36] Q. Zhang and M. Zhu. Li-Yau gradient bounds under nearly optimal curvature conditions. 2015. https://arxiv.org/pdf/1511.00791v2.pdf, [math.DG]. 